\title{
Polydipsia and autoshaping: Drinking and leverpressing as substitutes for eating
}

\author{
JAMES ALLISON and ROY MACK \\ Indiana University, Bloomington, Indiana
}

\begin{abstract}
Three experiments delivered food at fixed or random intervals independently of the rat's behavior, always less than the amount eaten with food freely available. The results revealed a polydipsic response to this experimental suppression of eating, and total drinking decreased as total eating increased. When we added a lever that signaled each food delivery, leverpressing and drinking rose far above their baseline levels; both responses decreased as total eating increased. When a similar schedule presented lever and food independently, rats still became polydipsic, but showed no sign of autoshaped leverpressing. A fourth experiment revealed a hypophagic response to schedules that suppressed drinking; total eating increased with total drinking. As mutual substitutes in the economic sense, one behavior falls as the other rises; as mutual complements in the economic sense, the two behaviors rise or fall together. We discuss polydipsia and autoshaping in terms of drinking as an intrinsic substitute for eating, and leverpressing as a learned substitute for eating. The results suggest a revision of conservation theory, which views drinking and eating as substitutes when the schedule suppresses eating but as complements when the schedule suppresses drinking.
\end{abstract}

Given small portions of dry food spaced several seconds apart, the rat may drink from a nearby water tube two or three times as much as it does with food available continuously (Falk, 1961). This excessive drinking, called "schedule-induced polydipsia," has inspired several theoretical accounts, none of which has yet gained widespread acceptance. As a wellspring of theoretical speculation, polydipsia rivals another example of excessive responding, called "autoshaping" (Brown \& Jenkins, 1968; Schwartz \& Gamzu, 1977) or "sign tracking" (Hearst \& Jenkins, 1974), studied mainly in pigeons, but also seen in rats. If we replace the water tube with a retractable lever and present the lever a few seconds before each delivery of food, the rat may press the lever much more than it would ordinarily, even though the delivery of food does not depend on pressing the lever (Peterson, 1972; Peterson, Ackil, Frommer, \& Hearst, 1972).

Conservation theory has seen some success in accounting for instrumental performance under a wide variety of response-contingent schedules (Allison, 1976, 1980, 1981b; Allison, Miller, \& Wozny, 1979; Shapiro \& Allison, 1978). This paper shows how conservation theory may also account for two kinds of excessive responding under noncontingent arrangements, schedule-induced polydipsia and autoshaped leverpressing. Experiment 1 tests a conservation

The research was supported by Grant MH34148 from the National Institute of Mental Health. We thank Gary Lucas for his comments on the manuscript. Requests for reprints should be sent to James Allison, Department of Psychology, Indiana University, Bloomington, Indiana 47405. model for the polydipsic response to a fixed-tim schedule that delivers free food at fixed periods 0 time. Experiment 2 extends the model to a settin that allows both autoshaping and polydipsia, random-time schedule that signals each delivery o food by presenting a lever $10 \mathrm{sec}$ in advance. Tw additional experiments reveal limits on the applica bility of the model.

\section{EXPERIMENT 1}

Copious research since Falk's discovery of the phe nomenon in 1961 has established several facts abou schedule-induced polydipsia and excluded several in terpretations (Falk, 1969, 1977; Staddon, 1977). Th classic pattern of excessive drinking occurs with grea reliability under a fixed- or variable-interval 60-se schedule that requires one leverpress for each 45-m food pellet over the course of a test session lasting 11 or more. The instrumental requirement plays no es sential role; polydipsia also occurs under fixed-tim schedules, which impose no instrumental requiremen (Burks, 1970). Within broad limits, the amount o drinking generally increases as the interval betwee food deliveries increases (Burks, 1970; Falk, 1966 Flory, 1971). As the amount of food per deliver rises, the polydipsia may decrease or disappear al together (Falk, 1967; Flory, 1971). Polydipsia ma: occur most readily if the rat's total food intake ha been held below the amount eaten when food is freel available (Falk, 1969). The excessive drinking canno be explained in terms of food as an accidental rein forcer of drinking, or drinking as an unconditione response to eating, to mention two of several hy 
potheses already advanced and discarded (Staddon, 1977; Stein, 1964).

We propose that most of the facts listed above can be handled by a simple linear equation:

$$
\mathrm{k}(\mathrm{NE})+\mathrm{U}_{\mathrm{d}}=\mathrm{kO}_{\mathrm{e}}+\mathrm{O}_{\mathrm{d}}
$$

In the context of Experiment 1, each side of the equation models a daily 2 -h period in which the rat gets all of the food and water it will get for the day. The right-hand side models a paired baseline session, with food and water freely available throughout, during which time the rat eats $\mathrm{O}_{e}$ grams of food and makes $\mathrm{O}_{\mathrm{d}}$ licks at the drinking tube. The left-hand side models a session in which a fixed-time schedule arranges a positive number of food deliveries, $\mathbf{N}$, spaced several seconds apart, with $\mathrm{E}$ grams per delivery; as in the baseline session, the drinking tube is freely available throughout. Thus, in the fixed-time session the schedule delivers a total of NE grams of food (NE $>0$ ), and the rat performs a total of $U_{d}$ licks at the drinking tube.

The $k$ that appears on both sides is a positive constant. Although other interpretations may be tenable, conservation theory interprets $\mathbf{k}$ in terms of some underlying dimension, physical or psychological, common to the two responses incorporated in the equation. For example, $k$ might refer to the amount of energy expended in eating $1 \mathrm{~g}$ of food, relative to the amount expended in making one lick at the drinking tube. Accordingly, each side would express, in licks, the total amount of energy allotted to eating plus drinking. The equation asserts that the rat conserves this total as between the fixed-time session on the left, and the baseline session on the right.

Rearrangement of Equation 1 shows some of the model's predictions more clearly:

$$
k\left(N E-O_{e}\right)+\left(U_{d}-O_{d}\right)=0 .
$$

By collecting like terms, Equation 2 compares the amount of food delivered in the fixed-time session with the amount eaten in the baseline session (NE $\mathrm{O}_{e}$ ) and the number of licks in the fixed-time session with the number performed in the baseline session $\left(U_{d}-O_{d}\right)$. Inspection of Equation 2 shows that if the fixed-time schedule delivers less food than the amount eaten in baseline $\left(\mathrm{NE}<\mathrm{O}_{e}\right)$, the rat should perform more licks than it did in baseline $\left(U_{d}>O_{d}\right)$. In other words, polydipsia occurs when the schedule suppresses eating experimentally, forcing the rat to eat less than it does when food and water are freely available.

The equation implies further that the polydipsic effect should increase with the amount by which the schedule suppresses eating; as $\mathrm{NE}$ decreases, $\mathrm{U}_{\mathrm{d}}$ should increase. We can decrease NE by decreasing $N$ or $\mathrm{E}$ independently. We can decrease $\mathrm{N}$, the number of deliveries, by increasing the time between deliveries over the 2-h session; we can decrease $\mathrm{E}$ by decreasing the amount of food per delivery. The literature suggests that each of these manipulations may cause some increase in polydipsic drinking, as the model implies.

The model predicts in addition that the total number of licks in the fixed-time session should decrease linearly as total food increases. Solving Equation 1 for $U_{d}$,

$$
\mathrm{U}_{\mathrm{d}}=\left(\mathrm{kO}_{\mathrm{e}}+\mathrm{O}_{\mathrm{d}}\right)-\mathrm{k}(\mathrm{NE}) \text {. }
$$

Equation 3 predicts that $U_{d}$, the total number of licks in the fixed-time session, will decrease linearly as the total grams of food, NE, increases. The intercept of the linear function is the baseline constant, $B=$ $\mathrm{kO}_{\mathrm{e}}+\mathrm{O}_{\mathrm{d}}$, and the slope is $-\mathrm{k}$. The intercept will rise with the number of licks performed in the paired baseline session $\left(\mathrm{O}_{d}\right)$ and with baseline food intake $\left(\mathrm{O}_{\mathrm{e}}\right)$. According to Equation 3, schedules that differ in the total number of deliveries, $\mathbf{N}$, and the amount of food per delivery, $\mathrm{E}$, should produce the same amount of drinking if they deliver the same amount of food, NE. Experiment 1 tested all of these implications.

Note that the model expressed as Equations 1-3 applies to schedules that deliver some food (NE $>0$ ), and has no implications for a "schedule" that delivers no food $(\mathrm{NE}=0)$. Readers who overlook this boundary condition may conclude mistakenly that the model predicts the greatest amount of drinking under a "schedule" that delivers no food. A no-food schedule would fall outside the scope of Equations 1-3 because conservation models relate the performance of responses under a schedule to performance of the same responses under a baseline condition in the absence of schedule constraints. The baseline terms in Equations 1-3 refer to a "paired" baseline condition that allows the rat to eat and drink without constraint because each of the schedules to be tested must allow substantial amounts of daily eating and drinking, enough to sustain the rat over the long period of daily testing that might be needed to achieve stable levels of performance. For a "schedule" that delivered no food, the appropriate baseline measurements would come under a "single" baseline condition that offered free access to water but no food. Because the "schedule" condition would be operationally identical to the single baseline condition, the model would make a totally vacuous prediction-identical amounts of drinking under the "two" conditions. For further discussion of issues concerning paired and single baselines, see Allison (1981b).

\section{Method}

Subjects. Six Sprague-Dawley rats, 90-day-old males purchased from Harlan Industries (Indianapolis, Indiana), served in the experiment. 
Apparatus. We tested the rats in three identical operant conditioning boxes, each controlled by a separate TRS-80 microcomputer. Accessible through a hinged Plexiglas door, the sheet metal interior measured $26 \mathrm{~cm}$ wide, $24 \mathrm{~cm}$ deep, and $16 \mathrm{~cm}$ high. A motorized shutter covered a circular hole in the wall opposite to the door. The hole was $2.5 \mathrm{~cm}$ wide, $5 \mathrm{~cm}$ from the left edge of the wall, and $9 \mathrm{~cm}$ above the floor. The shutter controlled access to a metal drinking tube with a 3-mm aperture, recessed $1 \mathrm{~cm}$ beneath the surface of the wall. An electronic drinkometer grounded to the sheet metal floor detected each discrete contact between tongue and tube. We recorded volumetric intake by reading water levels to the nearest milliliter on the $100-\mathrm{ml}$ reservoir, and corrected our readings for spillage by subtracting the amount collected in a catchment beneath the tube. A feeder mounted on top of the box delivered 45-mg food pellets (Bio-Mix T1300, manufactured by Bio-Serv, Inc., Frenchtown, N.J.) to a food trough located $6 \mathrm{~cm}$ above the floor and $13 \mathrm{~cm}$ to the right of the water tube. A retractable lever, not used in Experiment 1, projected through an oblong cutout between the tube and the trough, $9 \mathrm{~cm}$ above the floor. The lever stayed in its retracted position, flush with the surface of the wall, throughout Experiment 1. A noise generator masked extraneous sounds.

Procedure. For $S$ days before the first test session, each rat had free access to laboratory chow pellets and water in the home cage during the 2-h period reserved for its daily 2-h test sessions. Access to food and water was confined to that period for the duration of the experiment. One day before the first test session, each rat was adupted to its box for $15 \mathrm{~min}$, with food and water removed.

Daily tests began with at least seven baseline sessions that offered free access to the water tube and food $(25$ to $30 \mathrm{~g}$ of laboratory chow pellets placed on the floor). The baseline phase gave way to a period of training under six fixed-time schedules, and the experiment ended with another baseline phase. Each rat got all six schedules in an order determined by random assignment to a Latin square design. Each rat moved to the next phase or the next schedule when its total number of licks satisfied two stability criteria defined on a block of four consecutive sessions: no monotone change, and a standard deviation no greater than $15 \%$ of the block mean. The number of sessions needed to meet the stability criteria ranged from 7 to 21 in the first baseline phase (mean =12), 7 to 14 in the second (mean $=9$ ), and 4 to 12 in the fixed-time phase $(\operatorname{mean}=6)$.

Each fixed-time schedule delivered 1, 2, 3, or $445-\mathrm{mg}$ pellets at 60-sec or 90-sec intervals. Over the 2-h session, three 60-sec schedules made 120 deliveries of 1,2 , or 3 pellets per delivery, and three 90-sec schedules made 80 deliveries of 2,3 , or 4 pellets per delivery. Thus, the 60-sec schedules delivered a total of 5.4, 10.8, and $16.2 \mathrm{~g}$, and the $90-\mathrm{sec}$ schedules delivered $7.2,10.8$, and $14.4 \mathrm{~g}$. Within each delivery, pellets arrived about $1.5 \mathrm{sec}$ apart in the schedules that offered more than one pellet per delivery.

All of the rats urinated profusely during the fixed-time phase of the experiment. We discarded one rat that evaded our lick-detection system by licking its urine from the floor, a pattern seen in no other rat.

\section{Results and Dlscussion}

We based our analyses of performance under the fixed-time schedules on the mean of the criterion block of four consecutive sessions. Baseline performance refers to the mean of the two criterion blocks conducted before and after the fixed-time phase. (All five rats showed a slight rise in food and water intake between the first and the second baseline blocks.)

For all five individuals, each of the six schedules delivered less food than the amount eaten in baseline (baseline mean =19.9 g). According to Equation 2, this experimental suppression of eating should have produced a polydipsic effect under all six schedules. In agreement with the model, each rat, under each of the six schedules, exceeded both the baseline number of licks (mean $=4,246)$ and the baseline volumetric intake (mean $=25 \mathrm{ml}$ ). The fixed-time measures were nearly three times as large as the baseline measures in the most extreme cases.

In accord with Equation 3, total licks generally decreased as the total amount of food delivered by the fixed-time schedules increased $[F(5,20)=8.61, p<$ .0011 . This relation appears in Figure 1, which plots total licks against total food delivered (and eaten without fail) under the six schedules (filled circles) and the baseline condition (unfilled circle). Analysis of variance confirmed the strong linear trend apparent in Figure 1 and predicted by Equation 3 $[F(1,20)=41.05, p<.001]$. There was no significant curvilinear relation between total licks and total food delivered $[F(4,20)=.49]$. Because the rats ate 45-mg Bio-Serv pellets under the fixed-time schedules but laboratory chow pellets under the baseline condition, we felt some hesitation about including the basepoint (unfilled circle) in our trend analysis of the results shown in Figure 1. Nevertheless, we did our trend tests both ways, with and without the basepoint, and drew the same conclusions either way. The analysis that included the basepoint revealed a significant linear relation $[F(1,24)=73.51, p<.001]$ and no significant curvilinear relation $[F(S, 24)=.40] .^{1}$

The 90-sec, 3-pellet schedule delivered the same amount of food, $10.8 \mathrm{~g}$, as the 60-sec, 2-pellet schedule. As the model implies, these two schedules induced about the same number of licks $[t(4)=.47]$ and identical volumetric intakes $(51 \mathrm{ml})$. Only when the schedules differed in the total amount of food

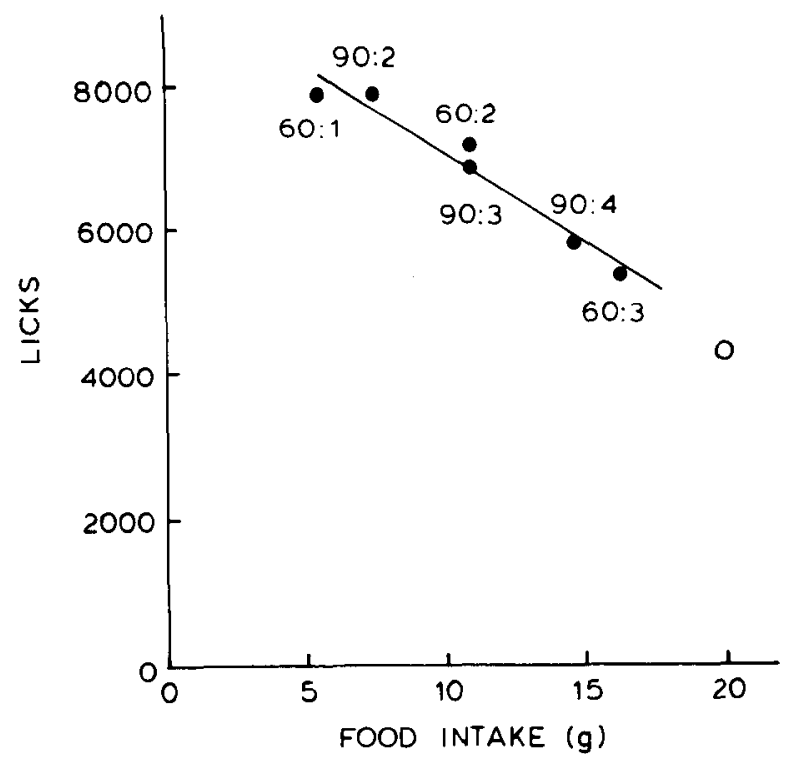

Figare 1. Total llets as a function of total food latake under the

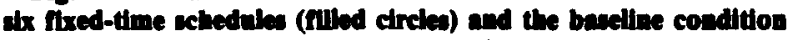
(unflled circle); group means, Experiment 1. The schedules dellvered 1, 2, 3, or 4 pellets at intervaly of 60 or 50 sec. 
delivered did the two familiar variables produce their two familiar effects: Holding constant the number of pellets per delivery, total licks increased with the time between deliveries; holding constant the time between deliveries, total licks decreased as pellets per delivery increased (see Figure 1).

Figure 1 shows a line fitted to the six fixed-time points by a linear regression analysis (licks $=9,527-$ $250 \mathrm{~g}, \mathrm{r}^{2}=.95$ ). Not shown in Figure 1 is a line fitted to the six fixed-time points plus the basepoint (licks = $9,671-265 \mathrm{~g}, \mathrm{r}^{2}=.97$ ).

As a final test of the model's ability to fit the quantitative details, we compared the model's prediction for the baseline constant, based entirely on performance under the fixed-time schedules, with values observed in the baseline condition. From the linear regression analysis of fixed-time performance, the value predicted for the group was $B=9,527$. If we use the same value of $\mathbf{k}$ for all individuals, the baseline constant observed for the individual rat is $\mathrm{B}=\mathrm{kO}_{\mathrm{e}}+$ $\mathrm{O}_{\mathrm{d}}=250 \mathrm{O}_{\mathrm{e}}+\mathrm{O}_{\mathrm{d}}$. Averaged across individuals, the value observed for $B, 9,214$, did not differ significantly from the value predicted $[t(4)=.48]$. We offer this comparison with a cautionary reminder. Recall that the rats ate 45-mg Bio-Serv pellets under the fixed-time schedules but laboratory chow pellets under the baseline condition. We feel fairly certain that if we had fed the rats Bio-Serv pellets in both conditions, the observed value of B would have risen slightly because of a slight rise in baseline food and water intake.

In summary, the results conformed reasonably well to predictions derived from the model. All six schedules induced the expected polydipsia; total licks decreased linearly as total food increased; different schedules that delivered the same total amount of food induced about the same amount of drinking; and baseline performance agreed with predictions derived from performance under the fixed-time schedules.

Further tests might well reveal some limitations. For example, we are not at all confident that $9 \mathrm{~g}$ of food, distributed in the form of $20045-\mathrm{mg}$ pellets spaced $36 \mathrm{sec}$ apart, would induce the same number of licks as three 3-g pellets spaced 2,400 sec apart. The model might also fail under prolonged testing with a schedule that offered too little daily food to sustain the rat, such as a schedule that made extremely small and infrequent deliveries. Nevertheless, our model and our results correct an oversight by calling attention to the total amount of food delivered by the schedule relative to the daily intake of free food, a potent variable often ignored in the theoretical analysis of schedule-induced polydipsia.

\section{EXPERIMENT 2}

In the original demonstration of autoshaping in the rat, Peterson (1972) used a variable-time 90-sec schedule to deliver 5095 -mg pellets per session, a total of $4.75 \mathrm{~g}$. His procedure signaled each forthcoming delivery by presenting a lever $15 \mathrm{sec}$ in advance, retracting the lever and delivering the food pellet simultaneously. After a few sessions, the rats far exceeded the pretreatment baseline level, about 10 presses/session, and performed some 200 to 300 presses/session toward the end of training. Peterson maintained his rats at $80 \%$ of their normal body weights by giving them supplemental feedings of 5 to $10 \mathrm{~g}$ daily. They had free access to water in the home cage, but no water in the test chamber-the standard practice of subsequent experiments on autoshaping with food delivery as the signaled event (Atnip, 1977; Locurto, Terrace, \& Gibbon, 1976; Stiers \& Silberberg, 1974).

Experiment 2 sought to produce autoshaping and polydipsia in the same experimental setting. Although several theorists view these two phenomena as adjunctive behaviors with similar origins, we know of no experiment in which the same procedure induced both types of behavior. Paradoxically, the theoretical model for our novel attempt implies that the addition of a drinking tube to the autoshaping paradigm might eliminate the autoshaping effect reported by Peterson (1972). Viewed in the context of our Experiment 1, the addition of a lever that signals the delivery of food might eliminate the polydipsic effects seen in Experiment 1.

We express the model for Experiment 2 as

$$
k(N E)+U_{d}+\mathrm{jU}_{\mathrm{p}}=\mathrm{kO}_{\mathrm{e}}+\mathrm{O}_{\mathrm{d}}+\mathrm{jO}_{\mathrm{p}}
$$

In the context of Experiment 2, the right-hand side models a 2-h baseline session, with food, water, and lever freely available throughout, in which the rat eats $\mathrm{O}_{e}$ grams of food, performs $\mathrm{O}_{d}$ licks at the drinking tube, and makes $O_{p}$ leverpresses. The lefthand side models a 2 -h session in which we make $\mathbf{N}=\mathbf{8 0}$ deliveries of food, $\mathbf{E}$ grams per delivery, on a random-time 90 -sec schedule that signals each delivery by presenting the lever $10 \mathrm{sec}$ in advance; $U_{d}$ refers to the number of licks in the random-time session, $U_{p}$ the number of leverpresses. In terms of conservation theory, the constant $k$ (or $j$ ) refers to the amount of the dimension entailed by eating $1 \mathrm{~g}$ (or making one leverpress), relative to the amount entailed by performing one lick at the tube. More neutrally, $k$ and $j$ have the status of scaling constants that allow us to fit three different responses into the same equation.

The paradox appears when we collect like terms,

$$
k\left(N E-O_{e}\right)+\left(U_{d}-O_{d}\right)+j\left(U_{p}-O_{p}\right)=0 .
$$

Because $k$ and $j$ are supposedly positive, Equation 5 implies that the experimental suppression of eating may cause any one of three effects that differ qualitatively. By suppressing eating experimentally, we 
assign a negative value to the first term on the left of Equation $5\left(\mathrm{NE}<\mathrm{O}_{\mathrm{e}}\right)$. To satisfy the equation, the rat must then respond to our experimental suppression of eating in such a way that the sum of the other two terms is positive. Thus, the rat can conform to the model by showing both effects, performing more than the baseline number of licks (polydipsia), and more than the baseline number of leverpresses (autoshaping). But the rat might also conform to the model by showing either effect in the absence of the other. In summary, the model allows polydipsia plus autoshaping, or either without the other, as permissible effects of the experimental suppression of eating.

The model for Experiment 1 implied a linear relation between total licks, $U_{d}$, and the total amount of food delivered by the schedules, NE. In contrast, the model for Experiment 2 implies that we should not necessarily see a linear relation between total licks and total food in Experiment 2. Solving Equation 4 for $U_{d}+j U_{p}$,

$$
\begin{aligned}
U_{d}+j U_{p} & =\left(k O_{e}+O_{d}+j O_{p}\right)-k(N E) \\
& =B^{\prime}-k(N E)
\end{aligned}
$$

Equation 6 predicts that the sum $U_{d}+j U_{p}$ will be linear in NE, but allows $U_{d}, U_{p}$, or both, to be nonlinear in NE. For example, consider three values of the independent variable, NE, spaced at equal intervals, such as 10,9 , and $8 \mathrm{~g}$. If these three schedules were to induce $1,000,4,000$, and 2,000 licks, and 10 , 20 , and 80 leverpresses, neither licks nor leverpresses would be linear in NE. Indeed, licks would not even be monotone in NE. But if $j=100$, the sum $U_{d}+j U_{p}$ would be linear in $N E$, because $U_{d}+j U_{p}=2,000$, 6,000 , and 10,000-linear in NE, as Equation 6 requires. To state its testable constraints more generally while making the weakest possible assumptions about $k$ and $j$, Equation 6 implies that there should exist some positive value of $j$ such that $U_{d}+j U_{p}$ decreases linearly as NE increases.

\section{Method}

Subject and Apparatus. We tested five Sprague-Dawley rats, 90-day-old males purchased from Harlan Industries, in the apparatus used in Experiment 1 . The metal lever, $3.5 \mathrm{~cm}$ wide and $1.3 \mathrm{~cm}$ thick, projected $2 \mathrm{~cm}$ into the box; a downward force on the end of the lever, about $.17 \mathrm{~N}$, closed a miniature switch and defined a leverpress.

Procedare. Except as noted, we used the general procedures described in Experiment 1. Baseline sessions conducted before and after the training phase offered free access to water, laboratory chow pellets, and the lever. We trained each rat with three random-time schedules, each of which made $\mathbf{8 0}$ food deliveries over the 2-h session at a mean interval of $90 \mathrm{sec}$; the computer selected each interval at random from an arithmetic series of five intervals $(30,60,90,120$, and $150 \mathrm{sec})$. The three schedules differed in the size of each delivery, 2, 3, or $445-\mathrm{mg}$ Bio-Serv pellets, for a total of $7.2,10.8$, or $14.4 \mathrm{~g}$. No two rats received the three schedules in the same order; of the six possible orders, we omitted only the one that started with the 4-pellet schedule and ended with the 2-pellet schedule.

The lever appeared $10 \mathrm{sec}$ before each delivery, and retracted when the feeder began the delivery. In addition to the stability criteria used in Experiment 1, we required no monotone change in total leverpresses across the four criterion sessions. The rats met the stability criteria in 9 to 17 sessions in the first baseline phase (mean $=15), 7$ to 14 in the second (mean =11), and 4 to 19 in the random-time phase (mean $=11)$.

\section{Results and Discussion}

Each of the three schedules suppressed eating by delivering less food than the individual ate under the baseline condition (baseline mean $=17.9 \mathrm{~g}$ ). In agreement with the model, each schedule induced in each of the five rats both more drinking and more leverpressing than seen in baseline (baseline means $=3,886$ licks, $23 \mathrm{ml}$, and 16 presses). The grand means for the training phase revealed significantly more licks in training $(14,575)$ than the baseline number, significantly more water consumption $(56 \mathrm{ml})$, and significantly more leverpresses (309) [smallest $F(1,12)=$ 21.72, $\mathrm{p}<.001]$.

Figure 2 plots total licks (top panel) and total leverpresses (middle panel) against the total amount of food delivered by the schedules (filled circles) and

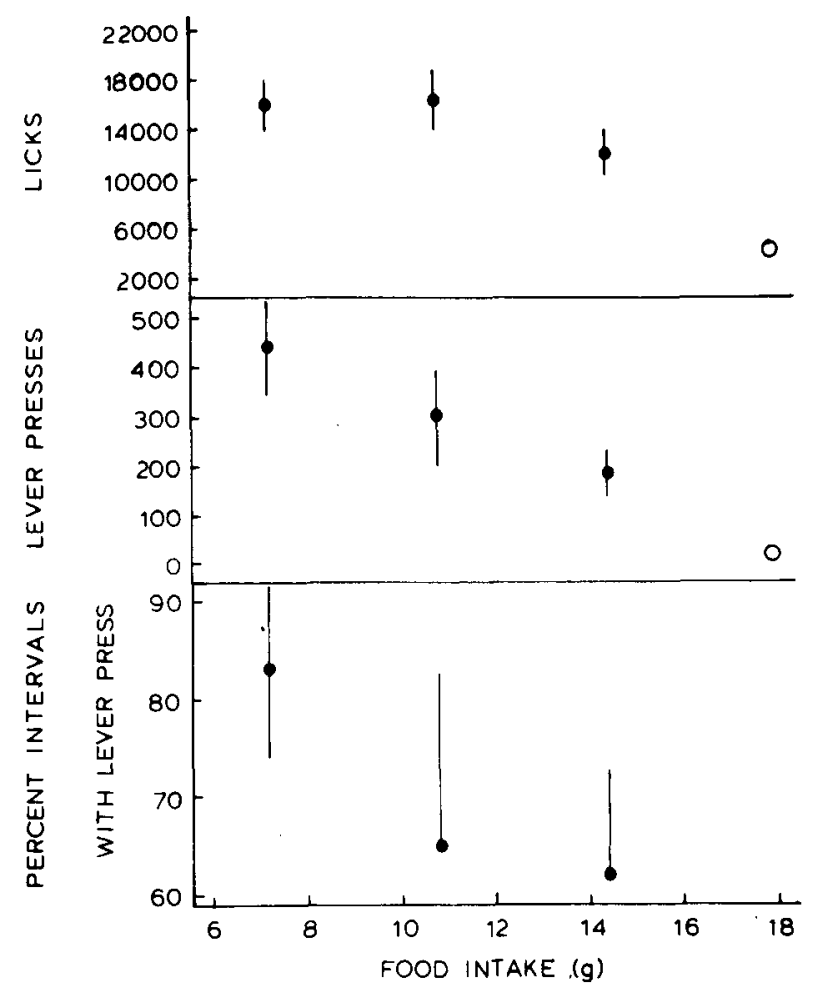

Figure 2. Total lleks (top panel), total leverpresses (middle panel), and percentage of intervalu with one or more leverpresace (bottom panel) as functions of total food intake under the three madom-time schedules (filled circles) and the baseline condition (unfilled clrcles); group means, Experiment 2. Each vertical line shows the standard error of the mean; standard errors of the basepoint means were too small to show graphicully. The achedules delivered 2,3, or 4 pellets at a mean interval of 90 sec. 
baseline food intake (unfilled circles). As in Experiment 1 , total licks decreased linearly as the schedule delivered more food $[F(1,8)=5.85, p<.05]$ and showed no significant curvilinearity $[F(1,8)=2.43$, p > .10]. Total leverpresses followed the same pattern, a linear decrease as the schedule delivered more food $[F(1,8)=14.93, p<.01]$, with no significant curvilinearity $[F(1,8)=.04]$. Four of the rats showed a steady decrease in total leverpresses as pellets per delivery rose from 2 to 3 , and from 3 to 4 ; the fifth rat performed its greatest number of leverpresses under the 2-pellet schedule, but its smallest under the 3-pellet schedule.

The bottom panel of Figure 2 shows that another measure of autoshaping, the percentage of intervals with one or more responses, agreed fairly well with total leverpresses. The percentage measure showed a marginally significant linear decrease as total food increased $[F(1,8)=5.01, p=.06]$, with no significant curvilinear trend $[F(1,8)=.86]$. On the percentage measure, all five rats responded more under the 2pellet schedule than under the 4-pellet schedule.

Figure 2 omits regression lines fitted to the schedule points. The equations of the lines were licks = $21,092-603$ g $\left(r^{2}=.71\right)$, presses $=697-36.0 \&\left(r^{2}=\right.$ $1.00)$, and percent intervals with leverpress $=101.5-$ $2.92 \mathrm{~g}\left(\mathrm{r}^{2}=.85\right)$. We also fitted regression lines to the three schedule points plus the basepoint: licks = $26,221-1,139 \mathrm{~g}\left(\mathrm{r}^{2}=.83\right)$ and presses $=727-39.1 \mathrm{~g}$ $\left(r^{2}=.99\right)$.

We can show that if total licks $\left(U_{d}\right)$ and total presses $\left(U_{p}\right)$ decrease linearly as food intake $(N E)$ increases, the sum $U_{d}+j U_{p}$ will also decrease linearly, as the model requires (see Equation 6 ). Let $U_{d}=a-$ $b(N E)$, and let $U_{p}=c-d(N E)$, as demonstrated by our statistical analysis. We wish to show that these two linear functions imply a third function in which $\mathrm{U}_{\mathrm{d}}+\mathrm{j} \mathrm{U}_{\mathrm{p}}$ decreases linearly as NE rises. If we multiply both sides of the second function by $j$ and add the result to the first function, we get $U_{d}+j U_{p}=(a+j c)$ $-(b+j d)(N E)$. Our result shows that if $U_{d}$ and $U_{p}$ both decrease linearly as $N E$ increases, so does $U_{d}$ $+\mathrm{jU}_{\mathrm{p}}$.

Iterative fits of the group means confirmed our impression that the data conformed closely to the model's implied constraints. These iterative fits showed that $\mathrm{U}_{\mathrm{d}}+\mathrm{j} \mathrm{U}_{\mathrm{p}}$ became progressively more linear in NE as we varied $j$ in several steps from a large negative value, through zero, to a large positive value. The $\mathbf{r}^{2}$ measure of linear fit, relatively small and insignificant when $j$ was negative or zero, increased steadily and approached 1 as $j$ approached 80.

Our inverse relation between leverpressing and the magnitude of food reward, though consistent with the conservation model, has no ready interpretation in other recent accounts of autoshaping and related phenomena. Killeen, Hanson, and Osborne (1978) explain adjunctive behavior in terms of the amount of arousal induced by the schedule. According to their account, large food deliveries spaced closely together in time should induce the greatest amount of arousal. Their measures of general activity supported this notion, but our measures showed the opposite relation, with large food deliveries spaced closely together inducing relatively little drinking (Experiment 1) or leverpressing (Experiment 2). Other theorists make no allowance for magnitude effects in the autoshaping paradigm. Thus, Jenkins, Barnes, and Barrera (1981) explain trial spacing effects in the autoshaping paradigm in terms of the average waiting time to food in the experimental setting relative to the average waiting time during the signal for food. In a similar analysis by Gibbon (1981), the expectancy of food depends in part on the amount of food per delivery, but the expectancy cancels out of Gibbon's expectancy ratio, a measure analogous to the relative waiting time of Jenkins et al. (1981).

Theories that attribute little importance to the amount of food per delivery in the autoshaping paradigm may do so because of a peculiar feature of the experimental literature on autoshaping. Most of that literature deals with the pigeon rather than the rat, and suggests that pigeons are relatively insensitive to the magnitude of the signaled event (Balsam, Brownstein, \& Shull, 1978). In contrast, the literature on response-contingent schedules contains many experiments with rats that reveal a strong inverse relation between instrumental responding and magnitude of reward, in agreement with conservation models for the experiments in question (Allison, 1980; Allison, Miller, \& Wozny, 1979).

In summary, the experimental suppression of eating in Experiment 2 produced a rise in each of two other behaviors, drinking from a water tube available continuously and pressing a lever presented shortly before each delivery of food. The inverse relation between total drinking and total eating seen in Experiment 1 appeared again in Experiment 2, along with a like relation between leverpressing and eating. Because the same manipulation produced both autoshaping and polydipsia, the results of Experiment 2 support the notion that these two examples of excessive responding under noncontingent arrangements have similar origins. In terms of the conservation model, each behavior rises above its baseline level because it has some substitute value for the behavior suppressed experimentally.

We designed Experiment 3 to examine the possibility that leverpressing and drinking might have different origins as substitutes. When both activities are available, the rat may find both leverpressing and drinking intrinsically substitutable for eating. Alternatively, leverpressing may have little or no intrinsic substitutability for eating; instead, the substitutability of leverpressing may arise from the role of the lever 
as a predictor of food. Experiment 3 tested these two alternatives.

\section{EXPERIMENT 3}

Experiment 2 signaled each food delivery by presenting the lever $10 \mathrm{sec}$ before each of the 80 randomtime deliveries. Experiment 3 also made 80 2-pellet deliveries on a random-time schedule, but eliminated the role of the lever as a predictor of food by presenting the lever independently of food on a random basis. Our new schedule presented the lever 80 times, $10 \mathrm{sec}$ each, on an independent random-time schedule. In effect, any particular food delivery and any particular lever presentation could be followed by either of two events: one of the remaining food deliveries or one of the remaining 10-sec lever presentations. If the substitutability of leverpressing for eating depends on the role of the lever as a predictor of food, our new schedule should produce appreciably less leverpressing than the corresponding schedule in Experiment 2.

\section{Method}

Sabjects and Appantus. We tested five rats, 90-day-old SpragueDawley males from Harlan Industries, in the apparatus used in Experiment 2.

Procedure. Except as noted, we used the same general procedures described for Experiment 2. Baseline phases with lever, water tube, and laboratory chow pellets freely available throughout each session preceded and followed a phase of training under a schedule that provided continuous access to the water tube while making 80 2-pellet food deliveries (45-mg Bio-Serv pellets) and 80 10-sec lever presentations independently of each other.

Each baseline session lasted exactly $2 \mathrm{~h}$. Training sessions averaged about $2 h$, but varied slightly about their 2 -h mean because the schedule selected each interevent interval at random. Specifically, the computer determined randomly at the beginning of the session, after each food delivery and after each lever presentation, whether the next event was to be one of the 80 food deliveries or one of the 80 lever presentations. At the same time, the computer determined at random how much time would elapse until the next event; in effect, the possibility set consisted of every integer value from 1 through $89 \mathrm{sec}($ mean $=45 \mathrm{sec})$. Thus, over a large number of random selections, the range of interevent intervals would converge on 1 to $89 \mathrm{sec}$, and the mean would converge on $45 \mathrm{sec}$. The mean interval between successive food deliveries would converge on $90 \mathrm{sec}$, as would the mean interval between successive lever presentations.

\section{Results and Discussion}

Like the corresponding schedule in Experiment 2, our new schedule induced a strong polydipsic effect; unlike Experiment 2, our new schedule produced no more than the baseline amount of leverpressing. Each of the five rats performed more licks under the schedule (mean $=10,816)$ than the baseline number [mean $=4,939 ; \mathrm{t}(4)=2.84, \mathrm{p}<.05$ ], and four of the five drank more under the schedule (mean $=39.2 \mathrm{ml}$ ) than in baseline [mean $=24.0 ; \mathrm{t}(4)=2.92, \mathrm{p}<.05$ ]. In contrast, the number of leverpresses observed under the schedule (mean $=2$ ) did not differ signifi- cantly from the baseline number $[$ mean $=10$; $t(4)=1.38, p>.20]$.

We conclude from the results of Experiments 2 and 3 that the rat may find drinking, but not leverpressing, intrinsically substitutable for eating. Only when the lever serves as a predictor of food does leverpressing have any appreciable substitutability for eating.

\section{EXPERIMENT 4}

In accounting for the polydipsic response to the schedules used in our first three experiments, the conservation model assumed that the extra drinking made up for the food shortage imposed by the schedule. Thus, the results supported the notion that drinking may have some value as a substitute for eating. It does not necessarily follow that eating may substitute for drinking: The dieter may accept plain lemon juice in place of a high-calorie salad dressing, but balk at lemonade made from olive oil and vinegar.

The conservation model expressed as Equation 1 makes no allowance for the possibility that eating might not substitute for drinking; indeed, the model even predicts a polyphagic response to a schedule that suppresses drinking. With a minor change in notation, we can modify the left-hand side of Equation 1 so as to model a schedule that allows free access to food, but only intermittent access to water. Defining $\mathbf{N}$ as the total number of accesses to the water tube, $\mathbf{D}$ as the number of licks allowed per access, and $U_{e}$ as total food intake under the schedule, Equation 1 becomes

$$
\mathrm{kU}_{\mathrm{e}}+\mathrm{ND}=\mathrm{kO}_{\mathrm{e}}+\mathrm{O}_{\mathrm{d}}
$$

Collecting like response terms to compare the amounts observed under baseline and schedule conditions,

$$
\mathrm{k}\left(\mathrm{U}_{\mathrm{e}}-\mathrm{O}_{\mathrm{e}}\right)+\left(\mathrm{ND}-\mathrm{O}_{\mathrm{d}}\right)=0 .
$$

Thus, Equation 8 predicts a polyphagic response to a schedule that suppresses drinking: If the schedule allows less than the baseline amount of drinking (ND < $\left.O_{d}\right)$, the rat should eat more than the baseline amount $\left(\mathrm{U}_{\mathrm{e}}>\mathrm{O}_{\mathrm{e}}\right)$. The model implies further that total eating should decrease linearly as total drinking increases; solving Equation 7 for $U_{e}$,

$$
\mathrm{U}_{\mathrm{e}}=\left[\left(\mathrm{kO}_{\mathrm{e}}+\mathrm{O}_{\mathrm{d}}\right)-\mathrm{ND}\right] / \mathrm{k} \text {. }
$$

Previous attempts to induce polyphagia through the intermittent delivery of water have met with no success (Myerson \& Christiansen, 1978; Wetherington \& Brownstein, 1979; Allen, Note 1). However, the 
reports of those attempts leave us uncertain that the schedules allowed less drinking than would have occurred under a baseline condition with free access to water and food. Experiment 4 closed this loophole in the experimental literature by comparing food intake under the baseline condition with intake under three schedules that allowed three different amounts of drinking, each less than the amount of drinking observed under the baseline condition.

\section{Method}

Subjects and Apparatus. We tested six Sprague-Dawley rats, 90 . day-old males from Harlan Industries, in a modified form of the apparatus used in Experiment 1 . The one modification replaced the food dispenser with a trough that allowed free access to 45mg Bio-Serv pellets.

Procedure. Except as noted, we used the same general procedures used in Experiment 1. All experimental sessions lasted $2 \mathrm{~h}$. The rat had free access to the Bio-Serv pellets and the water tube in baseline phases conducted before and after an intermediate phase of training under the three schedules, each of which presented the water tube on a fixed-time $90-\mathbf{s e c}$ schedule in an unlimited-hold procedure. Specifically, the three schedules arranged 5., 10-, or 20lick setups every $90 \mathrm{sec}(D=3,10$, or 20 licks). A 1-sec illumination of a white light mounted on the wall above the tube accompanied each presentation of the tube. If the rat failed to perform the $D$ licks in the setup, the tube remained available and licks that came due at the next setup were added to those unclaimed from the previous setup.

We tested each of the six rats under all three schedules in a Latin square design. One rat died before completing the experiment, and we omitted its data from the analysis.

\section{Results and Discussion}

Both total licks and total volumetric intake showed, without exception, that the five rats did a relatively large amount of drinking under schedules that allowed a relatively large number of licks per setup. As intended, the schedules suppressed drinking; each of the five rats performed fewer licks and took in less water under each of the three schedules than in the baseline condition.

Contrary to the model expressed as Equation 8, the rats displayed no sign of a polyphagic response to the schedules' suppression of their drinking. In direct opposition to the model, the data showed without exception that each schedule suppressed eating as well as drinking, and that the two responses decreased together. Inferential statistics showed all of the effects described above to be highly reliable.

Figure 3 plots total licks against total food intake (group means), and compares the results of Experiment 4 (squares) with those of Experiment 1 (circles). The two sets of results reveal a negative correlation between the two responses when the schedules suppressed eating (circles, Experiment 1), but a positive correlation when the schedules suppressed drinking (squares, Experiment 4). The results thus conformed to some familiar lore in the psychology of hunger and thirst: By drinking water, the rat can reduce some aspect of hunger, but cannot eat dry food without increasing its thirst.

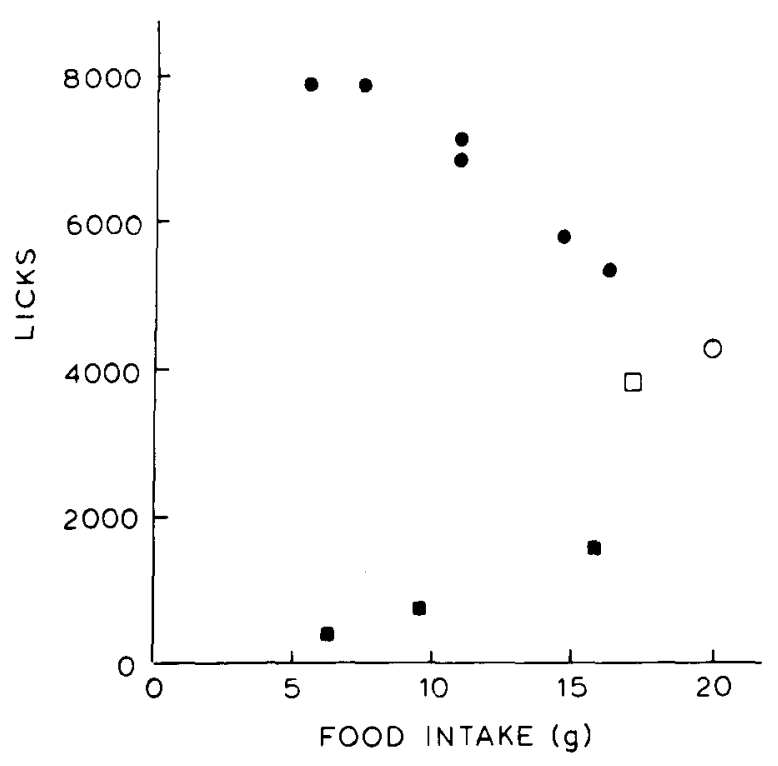

Figure 3. Total licks and total food intake in Experiment 1 (clrcles) and Experiment 4 (equarea), under the fixed-time schedules (filled symbols) and the baseline condition (unfilled symbols); group means. In Experiment 4, the schedules arranged 5., 10-, or 20-lick setups every 90 sac. In Experiment 1, the schedules dellivered $1,2,3$, or 4 pellets at intervals of 60 or 90 sec.

The results also contradicted a minimum deviation model for the two experiments (Staddon, 1979). According to the minimum deviation model, in performing under any sort of schedule, the organism will come as near as possible to the basepoint of the two responses controlled by the schedule. Because Experiment 1 placed no restriction on drinking, the number of licks performed under the schedules should have fallen on a horizontal line projected through the basepoint of eating and drinking. Instead, the rats performed significantly more than the baseline number of licks, rising above the line of minimum deviation (circles, Figure 3). Because Experiment 4 placed no restriction on eating, food intake under the schedules should have fallen on a vertical line projected through the basepoint. Instead, the rats ate significantly less than the baseline amount, falling to the left of the line of minimum deviation (squares, Figure 3). The minimum deviation model has similar difficulties in accounting for performance under concurrent ratio schedules and simple ratio schedules that offer an optional magnitude of responsecontingent food or water reward (Allison, 1981a).

The conservation model succeeded in Experiment 1, but failed in Experiment 4, because conservation theory assigns a positive numerical value to the dimensional constant $\mathbf{k}$. If $\mathbf{k}$ is positive, the model predicts a negative correlation between measures of drinking and eating; only if $\mathbf{k}$ could assume a negative value would the model predict the positive correlation seen in Experiment 4. With the help of some concepts from economics, we can fit the two experiments more successfully by revising the theory so as 
to recognize that $\mathbf{k}$ will have a positive value if the two behaviors function as substitutes, but negative if they function as complements.

To grasp the logic of our proposal, recall that $k$ tells how many units of one response are equivalent to one unit of the other response as the organism progresses toward the dimensional total specified by the equation. If the two responses are mutually substitutable, $\mathrm{k}$ will be positive. Thus, performance of either response will decrease the amount of the other response needed to reach the dimensional total allocated to the two responses together. For example, each cup of coffee (or tea) reduces the amount of additional tea (or coffee) needed to supply the desired amount of caffeine. Coffee and tea are substitutes. In contrast, two complementary goods (or responses) are consumed (or performed) in a fixed ratio to each other. For example, if we have less flour (or shortening), we will use less shortening (or flour) in our reduced production of bakery goods. Each time we find a left (or right) shoe, we need another right (or left) shoe to make a usable pair of shoes. Thus, if two different responses function as mutual complements, $\mathbf{k}$ will have a negative sign, because performance of either response will increase the amount of the other needed to reach the dimensional total. Finally, if the two responses are neither substitutes nor complements, $\mathrm{k}=0$.

By viewing $k$ as a measure of substitutability or complementarity, we can see that the sign of $k$ for a particular pair of responses might change from one setting to another. Imagine several cans of corn and several of peas, all cans identical in terms of size, weight, and shape. If the cans are to be used as building blocks for a 10-high stack, corn and peas are substitutes. Each time we add a can of corn (or peas) to the stack, we reduce by one the number of additional cans of peas (or corn) needed to complete the stack. But as ingredients in a recipe that calls for equal amounts of each, corn and peas are complements. For each can of one we open, we must open a can of the other.

According to this refined version of the theory, Experiment 1 revealed a positive value of $k$, and thereby showed that when the schedule suppresses eating, eating and drinking function as substitutes. Given less food, the rat drinks more water to attain a certain degree of hunger and thirst. In its motor and sensory components, including the sensations attendant on stomach distention, drinking may resemble eating closely enough to serve as a partial substitute for eating. Because Experiment 4 revealed a negative value of $k$, we would conclude that when the schedule suppresses drinking, eating and drinking function as complements. Given less water, the rat eats less dry food to attain a certain degree of hunger and thirst. Because bodily stores of water are a key ingredient in the chewing, swallowing, and digestion of dry food, the water deficit caused by the experi- mental suppression of drinking may make eating less food the way to maintain a tolerable balance, perhaps in defending the ratio of body water to lean body mass (Collier \& Levitsky, 1967). In summary, if the rat has plenty of water but not enough food, drinking and eating are substitutes; if the rat has plenty of food but not enough water, drinking and eating are complements.

We can appreciate the analysis more fully by repeating Equation 7, the original model for Experiment 4 , but assigning a negative value of $k$ :

$$
-\mathrm{kU}_{\mathrm{e}}+\mathrm{ND}=-\mathrm{kO}_{\mathrm{e}}+\mathrm{O}_{\mathrm{d}}
$$

We collect like terms to show the expected effect of schedules that suppress drinking,

$$
-\mathrm{k}\left(\mathrm{U}_{\mathrm{e}}-\mathrm{O}_{\mathrm{e}}\right)+\left(\mathrm{ND}-\mathrm{O}_{\mathrm{d}}\right)=0 \text {. }
$$

According to Equation 11, schedules that suppress drinking (ND $<\mathrm{O}_{d}$ ) will also suppress eating $\left(\mathrm{U}_{\mathrm{e}}<\right.$ $\mathrm{O}_{e}$ ). Solving Equation 10 for $U_{e}$, we see that the amount of eating performed under the schedule will increase linearly with the amount of drinking performed under the schedule. From $\mathrm{kU}_{\mathrm{e}}=\mathrm{ND}+\mathrm{kO}_{\mathrm{e}}$ $-O_{d}$,

$$
\left.\mathrm{U}_{\mathrm{e}}=\left[\mathrm{kO}_{\mathrm{e}}-\mathrm{O}_{\mathrm{d}}\right)+\mathrm{ND}\right] / \mathrm{k} \text {. }
$$

Thus, the amount of eating $\left(\mathrm{U}_{\mathrm{e}}\right)$ will increase linearly with the amount of drinking (ND). The function will rise with slope $1 / k$. The intercept, $O_{e}-\left(O_{d} / k\right)$, may be positive, zero, or negative.

In fitting Equation 12 to mean grams eaten under the three schedules used in Experiment 4 (closed squares, Figure 3), we found that performance under the three schedules fell neatly into line $[\mathrm{g}=3.09+.0079$ (licks), $r^{2}=1.00$ ]. A quantitative detail that escaped the model's grasp was the basepoint shown as the open square in Figure 3, which should have fallen in line with the three schedule points but actually fell far out of line. Still, all four points followed without exception the order prescribed by Equation 12.

Maybe the basepoints contain more error than the model. We might try to reduce error of measurement by including both basepoints in our analyses, with a cautionary reminder that the two experiments used different rats, and Experiment 1 used laboratory chow pellets rather than Bio-Serv pellets in the baseline measurement of food intake. Regression analysis of Experiment 4 shows that an upward line fits the three schedule points and the two basepoints reasonably well (licks $=-1,723+286 \mathrm{~g} ; \mathrm{r}^{2}=.84$ ). Applied to Experiment 1, the same analysis shows that a line with a comparable downward slope fits the six schedule points and the two basepoints reasonably well (licks $=9,888-296 \mathrm{~g} ; \mathrm{r}^{2}=.91$ ). Thus, two intersecting lines, one sloping upward, the other down, provide a parsimonious account of the data. 


\section{GENERAL DISCUSSION}

Our first three experiments show that we may understand polydipsia and autoshaping partly in terms of drinking and leverpressing as substitutes for eating. They suggest, in addition, two different kinds of substitutability. When the schedule suppresses eating, the rat seems to find drinking partly substitutable for eating; the substitutability appears to be intrinsic in the sense that it emerges with no further help from the experimenter, no external schedule that makes the chance to drink a statistical predictor of an imminent chance to eat (Experiments 1, 2, and 3). But when the schedule suppresses eating in the very same way, the rat may or may not find leverpressing partly substitutable for eating. This kind of substitutability appears to be extrinsic, in that it occurs when the lever predicts an imminent chance to eat (Experiment 2), but does not occur when lever and food appear independently of each other (Experiment 3). Perhaps the first kind of substitutability does not, and the second does, depend on associative learning.

We presume that the second kind of substitutability, the associative kind, plays a significant role in the organism's performance under response-contingent schedules, such as one that requires a certain amount of instrumental leverpressing for contingent access to food. In that type of schedule, the organism often responds to a higher instrumental requirement, or a smaller contingent reward, by performing a greater total amount of the instrumental benavior, and less of the contingent behavior (Allison et al., 1979). Conservation theory accounts for that sort of functional relation by assuming that the two responses act partly as substitutes, whether the functional relation appears under a response-contingent schedule or noncontingent schedules of the type used in Experiment 2.

The results also suggest that our conservation models cannot genuinely predict the results of such experiments without some advance understanding of the properties of the organism, the responses, and the experimental setting that determine whether the focal behaviors will function as mutual substitutes, complements, or neither. When we tried to induce hyperphagia by suppressing drinking, we assumed, in designing Experiment 4, that eating would substitute for drinking. When our procedure induced hypophagia instead, the results forced us to conclude that the suppression of drinking made complements of eating and drinking. In the meantime, perhaps we may view such experiments as a way of measuring substitutability and complementarity. But we see no contradiction between the model's descriptive and predictive uses. As a descriptive-analytic device applied to a set of existing data, the model offers a means of identifying behavioral substitutes and complements. Used in that fashion, the model constitutes a particular way of conceptualizing data, but only one among many possible ways. The same model can serve as a falsifiable predictor of new data in the hands of one who is prepared to state as an advance hypothesis whether each measured behavior be substitute, complement, or neither. As an example of its vulnerability, the original model for performance under fixed-ratio schedules has already undergone major revision in response to experimental data (Allison, 1981b). Unlike the original model, the 1981 version readily accounts for the curvilinear relation between instrumental and contingent responding sometimes seen with ratio schedules that cover an extremely broad range of instrumental response requirements.

\section{REFERENCE NOTE}

1. Allen, C. Patterns of activity and food oriented behavior. Unpublished manuscript, Arizona State University, 1980.

\section{REFERENCES}

Allison, J. Contrast, induction, facilitation, suppression, and conservation. Journal of the Experimental Analysis of Behavior, $1976,25,185-198$.

Allison, J. Conservation, matching, and the variable-interval schedule. Animal Learning \& Behavior, 1980, 8, 185-192.

Allison, J. Paired baseline performance as a behavioral ideal. Journal of the Experimental Analysis of Behavior, 1981, 35, 355-366. (a)

Allison, J. Economics and operant conditioning. In P. Harzem \& M. D. Zeiler (Eds.), Predictability, correlation, and contiguity. Chichester, England: Wiley, 1981. (b)

Alligon, J., Millen, M., \& Wozny, M. Conservation in behavior. Journal of Experimental Psychology: General, 1979, 108, 4-34.

AtnIP, G. W. Stimulus- and response-reinforcer contingencies in autoshaping, operant, classical, and omission training procedures in rats. Journal of the Experimental Analysis of Behavior, 1977, 28, 59-69.

Balsam, P. D., Brownstein, A. J., \& Shull, R. L. Effect of varying the duration of grain presentation on automaintenance. Journal of the Experimental Analysis of Behavior, 1978, 29, 27-36.

Brown, P. L., \& Jenkins, H. M. Auto-shaping of the pigeon's keypeck. Journal of the Experimental Analysis of Behavior, 1968, 11, 1-8.

Burks, C. D. Schedule-induced polydipsia: Are response-dependent schedules a limiting condition? Journal of the Experimental Analysis of Behavior, 1970, 13, 351-358.

Collien, G., \& Levitsky, D. Defense of water balance in rats: Behavioral and physiological responses to depletion. Journal of Comparative and Physiological Psychology, 1967, 64, 59-67.

FAlK, J. L. Production of polydipsia in normal rats by an intermittent food schedule. Science, 1961, 133, 195-196.

FaLK, J. L. Schedule-induced polydipsia as a function of fixed interval length. Journal of the Experimental Analysis of Behavior, 1966, 9, 37-39.

Falk, J. L. Control of schedule-induced polydipsia: Type, size, and spacing of meals. Journal of the Experimental Analysis of Behavior, 1967, 10, 199-206.

Falk, J. L. Conditions producing psychogenic polydipsia in animals. Annals of the New York Academy of Sciences, 1969, 157, 569-593.

FALK, J. L. The origin and functions of adjunctive behavior. Animal Learning \& Behavior, 1977, 5, 325-335.

FLORY, R. The control of schedule-induced polydipsia: Frequency 
and magnitude of reinforcement. Learning and Motivation, 1971, 2, 215-227.

Gibbon, J. The contingency problem in autoshaping. In C. M. Locurto, H. S. Terrace, \& J. Gibbon (Eds.), Autoshaping and conditioning theory. New York: Academic Press, 1981.

HenRst, E., \& Jenrins, H. M. Sign-tracking: The stimulusreinforcer relation and directed action. Austin, Tex: The Psychonomic Society, 1974.

Jenkins, H. M., Barnes, R. A., \& Barrera, F. J. Why autoshaping depends on trial spacing. In C. M. Locurto, H. S. Terrace, \& J. Gibbon (Eds.), Autoshaping and conditioning theory. New York: Academic Press, 1981.

Killeen, P. R., Hanson, S. J., \& Osborne, S. R. Arousal: Its genesis and manifestation as response rate. Psychological Review, 1978, 85, 571-581.

Locurto, C., Terrace, H. S., \& Gibbon, J. Autoshaping, random control, and omission training in the rat. Journal of the Experimental A nalysis of Behavior, 1976, 26, 451-462.

Myerson, J., \& Chribtiansen, B. Temporal control of eating on periodic water schedules. Physiology \& Behavior, 1979, 23, 279-282.

Peterson, G. B. Auto-shaping in the rat: Conditioned approach and contact behavior toward signals of food or brain-stimulation reinforcement. Unpublished doctoral dissertation, Indiana University, 1972.

Peterson, G. B., Ackil, J., Frommer, G. P., \& Hearst, E. Conditioned approach and contact behavior toward signals for food or brain-stimulation reinforcement. Science, 1972, 177, 1009-1011.

Schwartz, B., \& Gamzu, E. Pavlovian control of operant behavior: An analysis of autoshaping and its implications for operant conditioning. In W. K. Honig \& J. E. R. Staddon (Eds.), Handbook of operant behavior. Englewood Cliffs, N.J: PrenticeHall, 1977.
Shapiro, N., \& Allison, J. Conservation, choice, and the concurrent fixed-ratio schedule. Journal of the Experimental Analysis of Behavior, 1978, 29, 211-223.

Staddon, J. E. R. Schedule-induced behavior. In W. K. Honig \& J. E. R. Staddon (Eds.), Handbook of operant behavior. Englewood Cliffs, N.J: Prentice-Hall, 1977.

Staddon, J. E. R. Operant behavior as adaptation to constraint. Journal of Experimental Psychology: General, 1979, 108, 48-67.

Ste in, L. Excessive drinking in the rat: Superstition or thirst? Journal of Comparative and Physiological Psychology, 1964, 58, 237-242.

Stiers, M., \& Silberberg, A. Lever-contact responses in rats: Auto-maintenance with and without a negative responsereinforcer dependency. Journal of the Experimental Analysis of Behavior, 1974, 22, 497-506.

Wetherington, C. L., \& Brownstein, A. J. Schedule control of eating by fixed-time schedules of water presentation. Animal Learning \& Behavior, 1979, 7, 38-40.

\section{NOTE}

1. Significant linearity in the group data, with no significant curvilinearity, implies linearity among a substantial number of individual rats. We checked this implication by regressing total licks on total food intake for each individual. For each of the five rats, both regression constants had the proper sign, a positive intercept and a negative slope. The individual values of $\mathrm{r}^{2}$ ranged from .45 to .96 (mean $r^{2}=.77$, mean intercept $=9,532$, mean slope $=$ -256.28).

(Manuscript received December 15, 1981; revision accepted for publication May 19, 1982.) 